\title{
Prevalence of KRAS, BRAF, PI3K and EGFR mutations among Asian patients with metastatic colorectal cancer
}

\author{
LEE CHENG PHUA ${ }^{1}$, HUI WEN NG ${ }^{1}$, ANGIE HUI LING YEO ${ }^{1}$, ELYA CHEN ${ }^{2}$, MICHELLE SHU MEI LO ${ }^{2}$, \\ PEH YEAN CHEAH ${ }^{2-4}$, ERIC CHUN YONG CHAN ${ }^{1}, \mathrm{POH} \mathrm{KOON} \mathrm{KOH}^{2,5}$ and HAN KIAT HO ${ }^{1}$ \\ ${ }^{1}$ Department of Pharmacy, Faculty of Science, National University of Singapore, Singapore 117543; \\ ${ }^{2}$ Colorectal Cancer Research Laboratory, Department of Colorectal Surgery, Singapore General Hospital, Singapore 169856; \\ ${ }^{3}$ Saw Swee Hock School of Public Health, National University of Singapore, Singapore 117597; \\ ${ }^{4}$ Duke-NUS Graduate Medical School, National University of Singapore, Singapore 169857, Republic of Singapore
}

Received June 24, 2014; Accepted June 22, 2015

DOI: $10.3892 / 01.2015 .3560$

\begin{abstract}
Mutations in oncogenes along the epidermal growth factor receptor (EGFR) signaling pathway have been implicated in the resistance to cetuximab in patients with metastatic colorectal cancer (mCRC). However, the relative significance of these mutations based on their frequencies of occurrence in the Singaporean population remains unclear. In the present study, the prevalence of Kirsten rat sarcoma viral oncogene homolog (KRAS), v-Raf murine sarcoma viral oncogene homolog $\mathrm{B}$ $(B R A F)$, phosphoinositide 3-kinase $(P I 3 K)$ and EGFR somatic mutations were determined among Singaporean patients with mCRC. DNA extracted from 45 pairs of surgically resected tumor and normal mucosa samples was subjected to direct sequencing or restriction fragment length polymorphism. Associations of the genetic mutations with various clinicopathological parameters were further explored. Mutations in either codon 12 or 13 of KRAS were confirmed as prominent phenomena among the included Singaporean mCRC patients, at a prevalence comparable with that of Caucasian and patients of other Asian ethnicities [33.3\% (90\% confidence interval, 21.8-44.9\%)]. $K R A S$ mutation was not associated with clinicopathological
\end{abstract}

Correspondence to: Dr Han Kiat Ho, Department of Pharmacy, Faculty of Science, National University of Singapore, 18 Science Drive 4, Singapore 117543, Republic of Singapore

E-mail:phahohk@nus.edu.sg

Dr Poh Koon Koh, Colorectal Cancer Research Laboratory, Department of Colorectal Surgery, Singapore General Hospital, 20 College Road, Singapore 169856, Republic of Singapore

E-mail:kohpohkooncolorectal@gmail.com

${ }^{5}$ Present address: Capstone Colorectal Surgery Centre, Mt Elizabeth Medical Centre, 3 Mount Elizabeth, Singapore 228510, Republic of Singapore

Key words: colorectal cancer, metastatic, Kirsten rat sarcoma viral oncogene homolog gene, v-Raf murine sarcoma viral oncogene homolog B gene, phosphoinositide 3-kinase gene, epidermal growth factor receptor gene, mutation features, including age, gender and ethnicity of patients, or the tumor site, differentiation and mucinous status. Conversely, the prevalence of BRAF (0\%), PI3K (2.2\%) and EGFR $(0 \%)$ mutations were low. The results of the present study indicate that $K R A S$ mutations are prevalent among the studied population, and confirm the low prevalence of $B R A F, P I 3 K$ and $E G F R$ mutations. KRAS should be prioritized as an investigational gene for future studies of predictive biomarkers of cetuximab response among Singaporean patients with mCRC.

\section{Introduction}

Over the past few decades, the incidence of colorectal cancer (CRC) has escalated rapidly in Asian countries (1). In Singapore, $\mathrm{CRC}$ is the most commonly diagnosed cancer, accounting for 17.6 and $13.9 \%$ of cancers in males and females, respectively (2). The relatively high incidence of this disease has prompted efforts by clinicians and scientists to enhance the therapeutic management of CRC. Cetuximab (Erbitux ${ }^{\circledR}$ ) is a monoclonal antibody used widely in the targeted treatment of metastatic CRC (mCRC). It binds to the epidermal growth factor receptor (EGFR) and attenuates its downstream oncogenic signaling along the RAS/rapidly accelerated fibrosarcoma (RAF)/mitogen-activated protein kinase (MAPK) and phosphoinositide 3-kinase (PI3K)/AKT axes, thereby inhibiting tumor growth and progression (3). However, resistance to cetuximab remains a relevant issue. Studies have indicated that up to $80 \%$ of patients may incur additional treatment costs and skin toxicity without deriving a beneficial response from the treatment (4-6). For example, in patients with chemotherapy-refractory colorectal cancer whose tumors express EGFR, 9\% [95\% confidence interval (CI), 3-19\%] achieved a partial response. Toxicities such as an acne-like skin rash, predominantly on the face and upper torso, were experienced in $86 \%$ of the patients (6). In another study conducted on patients with irinotecan-refractory metastatic colorectal cancer, the rate of response to combination therapy of cetuximab plus irinotecan was $22.9 \%$, while that of cetuximab monotherapy was $10.8 \%$ (5). The identification of predictive markers of cetuximab response is therefore pertinent to improving the cost-effectiveness of the treatment and optimizing the quality of life for patients. 
Table I. Primers for polymerase chain reaction and gene sequencing.

\begin{tabular}{|c|c|c|c|}
\hline Gene & Primer sequence & Annealing temperature, ${ }^{\circ} \mathrm{C}$ & Product, bp \\
\hline \multicolumn{4}{|l|}{$K R A S$} \\
\hline \multirow[t]{2}{*}{ Exon 2} & Forward: 5'-GGTGGAGTATTTGATAGTGTATTAACC-3' & & \\
\hline & Reverse: 5'-AATGGTCCTGCACCAGTAATATG-3' & 60 & 246 \\
\hline \multirow[t]{2}{*}{ Exon 3} & Forward: 5'-TCTTTGGAGCAGGAACAATG-3' & & \\
\hline & Reverse: 5'-TGCATGGCATTAGCAAAGAC-3' & 55 & 402 \\
\hline \multicolumn{4}{|l|}{$B R A F$} \\
\hline \multirow[t]{2}{*}{ Exon 11} & Forward: 5'-TCCCTCTCAGGCATAAGGTAA-3' & & \\
\hline & Reverse: 5'-CGAACAGTGAATATTTCCTTTGAT-3' & 55 & 313 \\
\hline \multirow[t]{2}{*}{ Exon 15} & Forward: 5'-TCATAATGCTTGCTCTGATAGGA-3' & & \\
\hline & Reverse: 5'-GGCCAAAAATTTAATCAGTGGA-3' & 55 & 224 \\
\hline \multicolumn{4}{|l|}{ PI3K } \\
\hline \multirow[t]{2}{*}{ Exon 9} & Forward: 5'-GGGAAAAATATGACAAAGAAAGC-3' & & \\
\hline & Reverse: 5'-CTGAGATCAGCCAAATTCAGTT-3' & 55 & 250 \\
\hline \multirow{2}{*}{ Exon 20} & Forward: 5'-TTTGCTCCAAACTGACCAA -3' & & \\
\hline & Reverse: 5'-TGGAATCCAGAGTGAGCTTTC -3' & 55 & 349 \\
\hline \multicolumn{4}{|l|}{$E G F R$} \\
\hline \multirow[t]{2}{*}{ Exon 18} & Forward: 5'-GGCACTGCTTTCCAGCAT-3' & & \\
\hline & Reverse: 5'-CCCCACCAGACCATGAGA-3' & 60 & 248 \\
\hline \multirow[t]{2}{*}{ Exon 19} & Forward: 5'-CCCAGTGTCCCTCACCTTC-3' & & \\
\hline & Reverse: 5'-CCACACAGCAAAGCAGAAAC-3' & 60 & 239 \\
\hline \multirow[t]{2}{*}{ Exon 21} & Forward: 5'-TGATCTGTCCCTCACAGCAG-3' & & \\
\hline & Reverse: 5'-TCAGGAAAATGCTGGCTGAC-3' & 60 & 231 \\
\hline
\end{tabular}

In predicting the response of patients to anti-EGFR therapy, various genetic alterations along the EGFR pathway have emerged as promising markers. Landmark trials, including the multicenterCRYSTALand OPUS studies, have revealed that activating mutations in Kirsten rat sarcoma viral oncogene homolog $(K R A S)$, a critical regulatory protein along the RAS/RAF/MAPK axis, abrogate the therapeutic effect of cetuximab and serve as powerful negative predictors of its clinical efficacy (7-11). Therefore, major advisory bodies have promulgated restricting the administration of cetuximab to patients with $\mathrm{mCRC}$ and wild-type $K R A S$ status $(12,13)$. More recently, persuasive evidence has emerged for cetuximab resistance conferred by mutations in v-Raf murine sarcoma viral oncogene homolog $\mathrm{B}$ $(B R A F)$ and $P I 3 K$, regulators of the RAS/RAF/MAPK and PI3K/AKT pathways respectively $(14,15)$. Additionally, EGFR gene mutations, common features in non-small-cell lung cancer (NSCLC), have been linked to the efficacy of EGFR tyrosine kinase inhibitors including gefitinib (16-19). Given the similar mechanism of action of cetuximab and gefitinib, mutation at the EGFR tyrosine kinase domain could theoretically alter the sensitivity to cetuximab of mCRC.

While compelling data exists on the aforementioned mutations as potential predictive markers of cetuximab resistance in predominantly Caucasian patients with $\mathrm{mCRC}$, the relevance and importance of these findings within specific populations in Asia depends upon the local prevalence of these genetic alterations. Despite widespread efforts to establish the prevalence of these mutations among Asian countries, including China and Japan (20-26), there is scarce data regarding their prevalence in Singapore, a country with ethnic diversity comprising Chinese, Malay and Indian individuals. As ethnicity and lifestyle may influence mutation patterns (1), it is important to investigate and establish the prevalence of these genetic mutations among patients with mCRC in Singapore. A thorough review of the literature to date was conducted by searching the following keywords on PubMed in June 2014: 'KRAS OR K-Ras OR BRAF OR B-Raf OR PI3KCA OR PI3K-CA OR PI3K OR PIK3CA OR PIK3-CA OR PIK3 OR EGFR' AND 'colorectal cancer OR rectal cancer OR colon cancer' AND 'metastatic' AND 'Singapore'. The search revealed only one relevant study, which assessed KRAS mutations in eight mCRC tumors in Singapore (27). Furthermore, the frequencies of other genetic mutations relevant to the chemoresistance of cetuximab (BRAF, PIJK and EGFR) were not analyzed.

In order to establish this information, the present study aimed to comprehensively profile the frequencies of mutations in the hotspot regions of KRAS, BRAF, $P I 3 K$ and $E G F R$ in Singaporean patients with mCRC. The associations between the gene mutations and various clinicopathological characteristics were further examined. The understanding of their prevalence will help prioritize 
Table II. Mutational analysis methods for $K R A S, B R A F, P I 3 K$ and EGFR genes.

\begin{tabular}{lll}
\hline Gene & \multicolumn{1}{c}{ Mutations } & Analysis method \\
\hline KRAS & $\begin{array}{l}\text { Codon 12, 13 (Exon 2) } \\
\text { Codon 61 (Exon 3) }\end{array}$ & Gene sequencing \\
BRAF & $\begin{array}{l}\text { Codon 439, 459 (Exon 11) } \\
\text { Codon 600,601 (Exon 15) }\end{array}$ & Gene sequencing \\
PI3K & Codon 542, 545 (Exon 9) & Gene sequencing \\
& Codon 1043, 1047 (Exon 20) & \\
EGFR & G719S (Exon 18) & RFLP \\
& L858R (Exon 21) & RFLP \\
& Deletions (Exon 19) & Gene sequencing \\
\hline
\end{tabular}

$K R A S$, Kirsten rat sarcoma viral oncogene homolog; $B R A F$, v-Raf murine sarcoma viral oncogene homolog B; $P I 3 K$, phosphoinositide 3-kinase; $E G F R$, epidermal growth factor receptor; RFLP, restriction fragment length polymorphism.

Table III. Clinicopathological characteristics of 45 patients with metastatic colorectal cancer.

\begin{tabular}{lc}
\hline Characteristic & Value \\
\hline Age, years & \\
Mean & 59 \\
Range & $30-83$ \\
Gender, n (\%) & \\
Male & $29(64.4)$ \\
Female & $16(35.6)$ \\
Ethnicity, n $\%)$ & \\
Chinese & $34(75.6)$ \\
Malay & $7(15.6)$ \\
Indian & $4(8.9)$ \\
Tumor site, $\mathrm{n}(\%)$ & \\
Ascending colon & $1(2.2)$ \\
Hepatic flexure & $1(2.2)$ \\
Sigmoid colon & $21(46.7)$ \\
Rectosigmoid & $6(13.3)$ \\
Rectum & $16(35.6)$ \\
Tumor differentiation ${ }^{\mathrm{a}}, \mathrm{n}(\%)$ & \\
Moderate & $39(86.7)$ \\
Poor & $6(13.3)$ \\
\hline
\end{tabular}

aAll samples were moderately or poorly differentiated; no samples were well-differentiated.

investigationalgenes forfuturestudies of predictivebiomarkers of cetuximab response.

\section{Materials and methods}

Patients and tissue samples. Patients with mCRC (Dukes' Stage D) who underwent surgical tumor resection at the
Singapore General Hospital (Singapore) between June 2010 and October 2012 were included in the current study. The inclusion criteria were as follows: i) Histologically confirmed mCRC; ii) availability of sufficient amounts of tissue samples from the primary lesions for mutational analyses; and iii) availability of clinical information.

Paired tumor and mucosal tissues were snap-frozen in liquid nitrogen, microdissected and stored at $-80^{\circ} \mathrm{C}$ until analysis. Careful microdissection ensured that $\geq 90 \%$ of the tumor specimen comprised cancer cells. Matched normal mucosa samples were obtained $\geq 5 \mathrm{~cm}$ from the edges of the tumor. Clinicopathological parameters, including the age, gender and ethnicity of the patients, tumor site, degree of histological differentiation and histological type (mucinous or non-mucinous) were recorded. The study was approved by the Institutional Review Board of the Singapore General Hospital (2010/041/B) and informed consent was obtained from all participants.

DNA extraction and polymerase chain reaction (PCR) amplification. Genomic DNA was extracted from tissue samples using the QIAmp DNA Mini kit (Qiagen, Alameda, CA, USA), according to the manufacturer's instructions, and subjected to PCR to amplify KRAS exons 2 and 3, BRAF exons 11 and 15, $P I 3 K$ exons 9 and 20 and EGFR exons 18, 19 and 21. The primers used for PCR amplification were synthesized using First BASE Laboratories Sdn Bhd (Singapore) and are listed in Table I. These exons were selected for amplification as they encompass the mutational hotspots (Table II).

Each PCR reaction contained $300 \mathrm{ng}$ of genomic DNA, $2 \mu 1$ each of forward and reverse primers $(10 \mu \mathrm{M}), 20 \mu 1$ of $5 \mathrm{M}$ betaine (Sigma-Aldrich, St. Louis, MO, USA), $5 \mu 1$ of $2 \mathrm{mM}$ deoxynucleotide triphosphates, $2 \mu \mathrm{l}$ of $25 \mathrm{mM} \mathrm{MgSO}_{4}$ and $1 \mu \mathrm{l}$ of Novagen KOD Hot Start DNA polymerase (all from Merck Millipore, Tokyo, Japan) made up to a final volume of $50 \mu 1$. PCR cycling consisted of an initial denaturation at $94^{\circ} \mathrm{C}$ for 2 min, 35 cycles of denaturation at $94^{\circ} \mathrm{C}$ for $15 \mathrm{sec}$, primer annealing at 55 or $60^{\circ} \mathrm{C}$ (as stated in Table I) for $30 \mathrm{sec}$ and elongation at $68^{\circ} \mathrm{C}$ for $1 \mathrm{~min}$, followed by a final extension at $68^{\circ} \mathrm{C}$ for $5 \mathrm{~min}$. PCR products were then verified by $1.5 \%$ agarose gel electrophoresis (Sigma-Aldrich) and purified using a Multiscreen ${ }^{\circledR} \mathrm{PCR}_{\mu 96}$ plate (Merck Millipore, Carrigtwohill, Ireland) prior to either direct gene sequencing or restriction fragment length polymorphism (RFLP) analyses of the mutational hotspots (Table II) (28).

Gene sequencing. Purified PCR products of KRAS exons 2 and 3, BRAF exons 11 and 15,PI3K exons 9 and 20 and EGFR exon 19 were sequenced with BigDye ${ }^{\circledR}$ Terminator version 3.1 Cycle Sequencing kit (Applied Biosystems Life Technologies, Foster City, CA, USA) as per the manufacturer's instructions, and purified and analyzed with a 3730 ABI capillary electrophoresis system (Applied Biosystems Life Technologies). All sequencing reactions were performed using forward primers as stated in Table I, with the exception of PI3K exons 9 and 20 in which 5'-GGGAAAAATATGACAAAGAAAGCTATA-3' and 5'-TTGCTCCAAACTGACCAAAC-3' were used, respectively. DNA of normal mucosae from each patient was also amplified and sequenced alongside matched tumor DNA samples to rule out the occurrence of non-somatic mutations or polymorphisms. 
Table IV. Types of KRAS mutation detected in codons 12 and 13.

\begin{tabular}{llcl}
\hline KRAS exon 2 & Wild-type (amino acid) & Point mutation (amino acid) & Mutations, $\mathrm{n}(\%)$ \\
\hline Codon 12 & GGT $(\mathrm{G})$ & GAT (D) & $2(46.7)$ \\
& GGT (G) & GTT (V) & $1(6.7)$ \\
GGT (G) & AGT (S) & $1(6.7)$ \\
Codon 13 & GGT (G) & GCT (A) & $4(26.7)$ \\
\hline
\end{tabular}

Amino acids: G, Glycine; D, Aspartic acid; V, Valine; S, Serine; A, Alanine. KRAS, Kirsten rat sarcoma viral oncogene homolog.

Table V. Associations between KRAS mutation and clinicopathological characteristics.

\begin{tabular}{|c|c|c|c|c|}
\hline Characteristic & All $(n=45)$ & $K R A S$ wild-type $(\mathrm{n}=30)$ & $K R A S$ mutant $(\mathrm{n}=15)$ & P-value \\
\hline Age $($ mean $\pm \mathrm{SD})$, years ${ }^{\mathrm{a}}$ & 59 & $56.6 \pm 10.2$ & $64.5 \pm 8.9$ & 0.013 \\
\hline \multicolumn{5}{|l|}{ Gender, n $(\%)^{\mathrm{b}}$} \\
\hline Male & 29 & $16(55.2)$ & $13(44.8)$ & \multirow[t]{2}{*}{0.028} \\
\hline Female & 16 & $14(87.5)$ & $2(12.5)$ & \\
\hline \multicolumn{5}{|l|}{ Ethnicity, $\mathrm{n}(\%)^{\mathrm{c}}$} \\
\hline Chinese & 34 & $23(67.6)$ & $11(32.4)$ & \multirow[t]{3}{*}{0.137} \\
\hline Malay & 7 & $6(85.7)$ & $1(14.3)$ & \\
\hline Indian & 4 & $1(25.0)$ & $3(75.0)$ & \\
\hline \multicolumn{5}{|l|}{ Tumor site, $\mathrm{n}(\%)^{\mathrm{b}}$} \\
\hline Colon & 29 & $22(75.9)$ & $7(24.1)$ & \multirow[t]{2}{*}{0.078} \\
\hline Rectum & 16 & $8(50.0)$ & $8(50.0)$ & \\
\hline \multicolumn{5}{|c|}{ Tumor differentiation, $\mathrm{n}(\%)^{\mathrm{c}}$} \\
\hline Moderate & 39 & $29(74.4)$ & $10(25.6)$ & \multirow[t]{2}{*}{0.012} \\
\hline Poor & 6 & $1(16.7)$ & $5(83.3)$ & \\
\hline \multicolumn{5}{|l|}{ Histological type, $\mathrm{n}(\%)^{\mathrm{c}}$} \\
\hline Mucinous & 6 & $2(33.3)$ & $4(66.7)$ & \multirow[t]{2}{*}{0.157} \\
\hline Non-mucinous & 39 & $28(71.8)$ & $11(28.2)$ & \\
\hline
\end{tabular}

Obtained by aindependent t-test, ${ }^{\mathrm{b}} \chi^{2}$ test or ${ }^{\mathrm{c}}$ Fisher's exact test. KRAS, Kirsten rat sarcoma viral oncogene homolog; SD, standard deviation.

RFLP analysis. The presence of G719S (EGFR exon 18) and L858R (EGFR exon 21) mutations were determined by RFLP analyses using restriction endonucleases DdeI and Sau96I (New England Biolabs, Inc., Singapore) (29), respectively. Purified PCR product $(15 \mu \mathrm{l})$ was digested with 10 units of DdeI or Sau96I in a total volume of $20 \mu 1$ at $37^{\circ} \mathrm{C}$ for $2 \mathrm{~h}$, and electrophoresed through a $2.5 \%$ agarose gel. Upon digestion by restriction enzyme $D d \mathrm{eI}$, the wild-type allele of EGFR exon 18 produced fragments at 27 and $221 \mathrm{bp}$ while the mutant G719S allele yielded fragments at 27, 92 and $129 \mathrm{bp}$. The SW48 human colorectal adenocarcinoma cell line (American Type Culture Collection, Manassas, VA, USA), which harbors a heterozygous G719S mutation (30), was run alongside as a positive control. Upon digestion by Sau96I, the wild-type allele of EGFR exon 21 yielded fragments at 55 and $176 \mathrm{bp}$, while the mutant L858R allele produced three fragments $(55,86$ and $90 \mathrm{bp})$.

Statistical analysis. The normal approximation method was used to construct a $90 \%$ CI in estimating the prevalence of genetic mutation. This conservative CI was used due to the small sample size. Associations of genetic mutations with clinicopathological parameters, including gender, ethnicity, tumor location, tumor differentiation and histological type were explored using the $\chi^{2}$ or Fisher's exact tests (SPSS version 16; SPSS Inc, Chicago, IL, USA). Associations with age were evaluated using an independent samples Student's t-test. A Bonferroni correction for multiple testing was performed by dividing the critical $\mathrm{P}$-value $(\mathrm{P}=0.05)$ by the number of comparisons being made $(n=6)$. Therefore, statistical significance was established at $\mathrm{P}<0.008$.

\section{Results}

Patient characteristics. A total of 45 patients with mCRC, comprising the major ethnic groups in Singapore (34 Chinese, 7 Malay and 4 Indian patients) and reflecting their prevailing population distribution were enrolled into the study. Tumors were located predominantly in the sigmoid colon (46.7\%), 


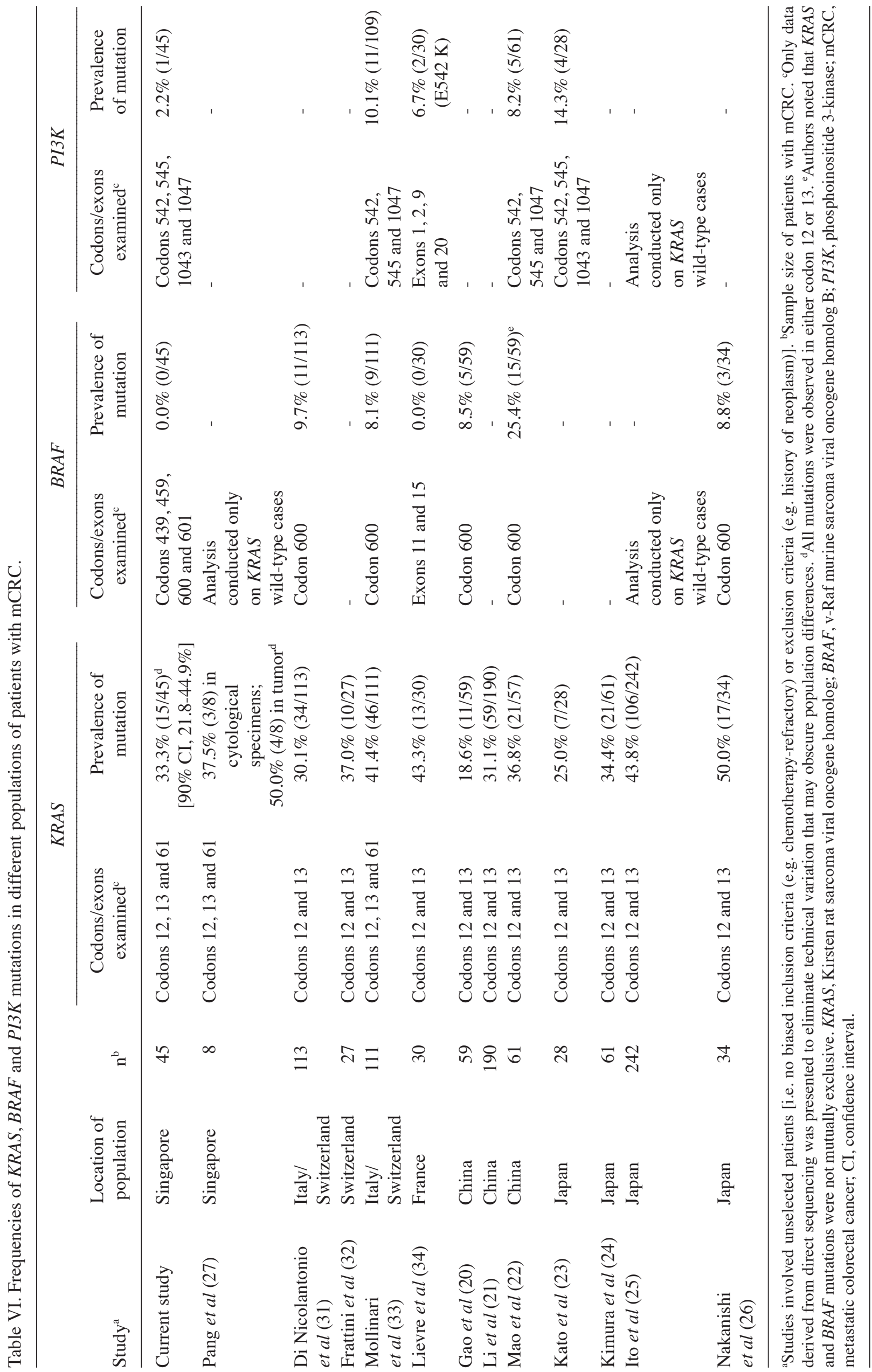


rectum (35.6\%) and rectosigmoid region (13.3\%) and were moderately or poorly differentiated. Table III summarizes the clinicopathological characteristics of the recruited patients.

KRAS mutational profiling. Tumor KRAS mutation was identified in 15 patients, equal to a prevalence of $33.3 \%(90 \% \mathrm{CI}$, 21.8-44.9\%). In addition, 11 mutations (73.3\%) were identified in codon 12, while 4 mutations occurred in codon 13 (26.7\%). The types of gene mutations detected in KRAS are tabulated in Table IV. The most frequently observed mutation was a GGT>GAT transition (G12D). By contrast, no mutations were detected in codon 61 of exon 3. No normal mucosae exhibited any mutations, indicating that all tumor mutations were somatic in nature.

Correlation of KRAS gene mutations with clinicopathological characteristics. No statistically significant differences were identified in terms of age, gender, ethnicity, tumor site, tumor differentiation and mucinous status between patients with and without KRAS mutations $(\mathrm{P}>0.008$; Table $\mathrm{V})$.

PI3K mutational profiling. Of the 45 tumor samples, only one sample (2.2\%) harbored a somatic mutation of the PI3K gene. The observed $P I 3 K$ mutation was a heterozygous GAG $>$ GCG transversion in codon 545 of exon 9 (E545A), and was identified in a sigmoid colonic tumor displaying KRAS wild-type, resected from a 30-year-old female patient of Chinese ethnicity (the youngest patient in the cohort).

$B R A F$ and EGFR mutational profiling. No mutations (0/45 samples) were detected in codons 439, 459, 600 and 601 of the $B R A F$ gene. Similarly, all samples exhibited wild-type status at codons 719 and 858 of the EGFR gene. No deletion mutations were observed at $E G F R$ exon 19.

\section{Discussion}

Mutations in KRAS, BRAF and PI3K, encoding the key regulatory proteins downstream of EGFR, play vital roles in colorectal carcinogenesis and have been closely linked with clinical resistance to cetuximab (7-11,14-15). To further elucidate the importance of these genetic alterations in the context of Singaporean mCRC, their currently undefined local prevalence was characterized in the present study. The results revealed a substantial occurrence of KRAS mutations, the frequency of which (33.3\%) resembled that in north Asian (e.g. Chinese and Japanese) and Caucasian populations of mCRC patients (20-50\%) (20-26,31-34). For comparison, representative studies from Japan, China and Europe, in which direct sequencing of KRAS were conducted at similar codons, are summarized in Table VI. The substantial prevalence of KRAS mutations provides a strong basis for future investigations on its utility as a predictor of cetuximab efficacy in the Singaporean population. Notably, the observed mutations were located exclusively in codons 12 and 13 of exon 2 , consistent with reports on its predominance (90\%) in exon 2 and infrequent occurrence at codon 61 of exon 3 (35). It is also noteworthy that codons 12 and 13 of exon 2 encode for two adjacent glycine residues situated in close proximity to the catalytic site of KRAS. Mutations of these codons abolish the intrinsic guanosine triphosphatase activity of the KRAS protein (35), leading to its constitutive activation and tumor growth. Within exon 2, the distribution of mutations between codons 12 and 13 was also congruent with prior reports of patients with mCRC, in which $70 \%$ of mutations occurred at codon $12(20,32,33)$. In addition, G12D (GGT>GAT) was revealed to be the most prominent mutation type, in concordance with evidence from Chinese and Caucasian mCRC patients $(21,22,32)$. Taken together, codons 12 and 13 represent potential subjects of interest for future Singapore-based studies investigating the role of KRAS mutation status in predicting treatment response.

A number of studies conducted in Caucasian and Asian CRC populations found no association between the prevalence of KRAS mutations and various clinicopathological parameters, including the gender and age of patients as well as tumor location, histological type and differentiation (23,26,36-38). Analogous findings were also evident among mCRC patients from Asian populations (39). Similarly, the various clinicopathological parameters of Singaporean patients with mCRC investigated in the present study did not correlate significantly with the occurrence of KRAS mutations. Such poor correlation between genotype and phenotype is not unexpected, as CRC is a heterogeneous disease defined by host genetic, environmental, nutritional and gut microbial factors (40).

The present study also revealed an extremely low prevalence of $B R A F$ and $P I 3 K$ mutations. Encoding a downstream effector of KRAS in the MAPK pathway, $B R A F$ has also has been studied extensively with regard to CRC. The $B R A F$ V600E mutation has been documented to occur at a lower rate $(0-10 \%)$ than KRAS mutations in Caucasian and Asian mCRC patients $(20,31,33,34)$ (Table VI). This observation was reflected in the present study, in which no $B R A F$ mutations were detected. By contrast, the mutation rate of the gene encoding PI3K (2.2\%), a regulator of PI3K/AKT signaling, appeared to be marginally lower compared with that of Chinese and Caucasian mCRC populations $(\sim 10 \%)(22,23,33,34)$ (Table VI). The low observed frequency of PI3K mutations may possibly be explained by environmental influences, such as diet and lifestyle, or a difference in hotspot codons in Singaporean patients.

As $E G F R$ gene mutation has been a crucial determinant of the sensitivity of NSCLC to EGFR tyrosine kinase inhibitors, it was of interest to determine its mutation rate in patients with mCRC. In the present study, however, neither missense (G719S in exon 18 and L858R in exon 21) nor deletion mutations (in exon 19) were identified. Specifically, although the EGFR G719S mutation, an NSCLC-relevant somatic mutation, was previously discovered in the SW48 colon cancer cell line (30), the present data suggested this mutation was not clinically prevalent in the context of mCRC. The paucity of EGFR somatic mutations in Singaporean patients with mCRC was consistent with findings in their Caucasian counterparts (28). This highlights the presence of a different set of genetic alterations that drives the progression of mCRC compared with NSCLC. Considering that the EGFR activating gene mutation is responsible for the sensitivity of NSCLC to EGFR tyrosine kinase inhibitors, the non-existence of activating EGFR mutations may also explain the general lack of response towards anti-EGFR therapy in mCRC. Nevertheless, with regard to predicting the efficacy of cetuximab therapy, the present 
analyses demonstrated collectively that $B R A F, P I 3 K$ and $E G F R$ mutations assume less significant roles, owing to their rarity of occurrence, compared with that of KRAS among Singaporean $\mathrm{mCRC}$ patients.

In conclusion, the frequencies of $K R A S, B R A F, P I 3 K$ and $E G F R$ mutations were determined in the Singaporean mCRC population, and KRAS mutations were confirmed to be prominent phenomena. The present study thereby lays the foundation for future investigations into predictive biomarkers of cetuximab response, and represents an important step towards personalized medicine for the local Singaporean mCRC population.

\section{Acknowledgements}

The authors would like to thank Ms. Wei Lin Goh, Ms. Grace Yu Hui Wong and Dr Tony Kiat Hon Lim (Singapore General Hospital, Singapore) for their technical expertise and logistical support. This project was funded by Merck Pte Ltd., the Singapore Ministry of Education's academic research grants (nos. R-148-000-133-112 and R-148-000-193-112) and the NUS President Graduate Fellowship.

\section{References}

1. Sung JJ, Lau JY, Goh KL and Leung WK; Asia Pacific Working Group on Colorectal Cancer: Increasing incidence of colorectal cancer in Asia: implications for screening. Lancet Oncol 6: 871-876, 2005

2. Singapore cancer registry interim report: Trends in cancer incidence in Singapore 2007-2011. In: National Registry of Diseases Office (NRDO), Health Promotion Board, Singapore, 2011.

3. Vecchione L, Jacobs B, Normanno N, et al: EGFR-targeted therapy. Exp Cell Res 317: 2765-2771, 2011.

4. Jonker DJ, O'Callaghan CJ, Karapetis CS, et al: Cetuximab for the treatment of colorectal cancer. N Engl J Med 357: 2040-2048, 2007.

5. Cunningham D, Humblet Y, Siena S, et al: Cetuximab monotherapy and cetuximab plus irinotecan in irinotecan-refractory metastatic colorectal cancer. N Engl J Med 351: 337-345, 2004.

6. Saltz LB, Meropol NJ, Loehrer PJ Sr, et al: Phase II trial of cetuximab in patients with refractory colorectal cancer that expresses the epidermal growth factor receptor. J Clin Oncol 22 : 1201-1208, 2004.

7. Karapetis CS, Khambata-Ford S, Jonker DJ, et al: K-ras mutations and benefit from cetuximab in advanced colorectal cancer. $\mathrm{N}$ Engl J Med 359: 1757-1765, 2008.

8. Van Cutsem E, Lang I, D'haens G, et al: Kras status and efficacy in the first-line treatment of patients with metastatic colorectal cancer $(\mathrm{mcrc})$ treated with folfiri with or without cetuximab: The crystal experience. J Clin Oncol, 2008 ASCO Annual Meeting Proceedings 26: 2, 2008.

9. Van Cutsem E, Köhne CH, Láng I, et al: Cetuximab plus irinotecan, fluorouracil and leucovorin as first-line treatment for metastatic colorectal cancer: Updated analysis of overall survival according to tumor KRAS and BRAF mutation status. J Clin Oncol 29: 2011-2019, 2011.

10. Bokemeyer C, Bondarenko I, Hartmann JT, De Braud FG, Volovat C, Nippgen J, Stroh C, Celik I and Koralewski P: Kras status and efficacy of first-line treatment of patients with metastatic colorectal cancer (mcrc) with folfox with or without cetuximab: The opus experience. J Clin Oncol 26 (Suppl 4000): 2008.

11. Bokemeyer C, Bondarenko I, Hartmann JT, de Braud F, Schuch G, Zubel A, Celik I, Schlichting M and Koralewski P: Efficacy according to biomarker status of cetuximab plus FOLFOX-4 as first-line treatment for metastatic colorectal cancer: The OPUS study. Ann Oncol 22: 1535-1546, 2011.

12. Allegra CJ, Jessup JM, Somerfield MR, et al: American Society of Clinical Oncology provisional clinical opinion: Testing for KRAS gene mutations in patients with metastatic colorectal carcinoma to predict response to anti-epidermal growth factor receptor monoclonal antibody therapy. J Clin Oncol 27: 2091-2096, 2009.
13. U.S. Food and Drug Administration: Class labeling changes to anti-EGFR monoclonal antibodies, cetuximab (erbitux) and panitumumab (vectibix): Kras mutations. 2009. http://www.fda. gov/AboutFDA/CentersOffices/OfficeofMedicalProductsandTobacco/CDER/ucm172905.htm. Accessed July 152013.

14. Laurent-Puig P, Cayre A, Manceau G, et al: Analysis of PTEN, BRAF and EGFR status in determining benefit from cetuximab therapy in wild-type KRAS metastatic colon cancer. J Clin Oncol 27: 5924-5930, 2009.

15. De Roock W, Claes B, Bernasconi D, et al: Effects of KRAS, BRAF, NRAS and PIK3CA mutations on the efficacy of cetuximab plus chemotherapy in chemotherapy-refractory metastatic colorectal cancer: A retrospective consortium analysis. Lancet Oncol 11: 753-762, 2010.

16. Pao W, Miller V, Zakowski M, et al: EGF receptor gene mutations are common in lung cancers from 'never smokers' and are associated with sensitivity of tumors to gefitinib and erlotinib. Proc Natl Acad Sci USA 101: 13306-13311, 2004.

17. Lynch TJ, Bell DW, Sordella R, et al: Activating mutations in the epidermal growth factor receptor underlying responsiveness of non-small-cell lung cancer to gefitinib. N Engl J Med 350: 2129-2139, 2004.

18. Eck MJ and Yun CH: Structural and mechanistic underpinnings of the differential drug sensitivity of EGFR mutations in non-small cell lung cancer. Biochim Biophys Acta 1804: 559-566, 2010.

19. Takano T, Ohe Y, Sakamoto H, et al: Epidermal growth factor receptor gene mutations and increased copy numbers predict gefitinib sensitivity in patients with recurrent non-small-cell lung cancer. J Clin Oncol 23: 6829-6837, 2005.

20. Gao J, Wang TT, Yu JW, Li YY and Shen L: Wild-type kras and braf could predict response to cetuximab in chinese colorectal cancer patients. Chin J Cancer Res 23: 271-275, 2011.

21. Li FH, Shen L, Li ZH, Luo HY, Qiu MZ, Zhang HZ, Li YH and Xu RH: Impact of KRAS mutation and PTEN expression on cetuximab-treated colorectal cancer. World J Gastroenterol 16: 5881-5888, 2010

22. Mao C, Zhou J, Yang Z, Huang Y, Wu X, Shen H, Tang J and Chen Q: KRAS, BRAF and PIK3CA mutations and the loss of PTEN expression in Chinese patients with colorectal cancer. PLoS One 7: e36653, 2012.

23. Kato S, Iida S, Higuchi T, Ishikawa T, Takagi Y, Yasuno M, Enomoto M, Uetake $\mathrm{H}$ and Sugihara K: PIK3CA mutation is predictive of poor survival in patients with colorectal cancer. Int J Cancer 121: 1771-1778, 2007.

24. Kimura T, Okamoto K, Miyamoto H, et al: Clinical benefit of high-sensitivity KRAS mutation testing in metastatic colorectal cancer treated with anti-EGFR antibody therapy. Oncology 82: 298-304, 2012.

25. Ito Y, Yamada Y, Asada K, Ushijima T, Iwasa S, Kato K, Hamaguchi T and Shimada Y: EGFR L2 domain mutation is not correlated with resistance to cetuximab in metastatic colorectal cancer patients. J Cancer Res Clin Oncol 139: 1391-1396, 2013.

26. Nakanishi R, Harada J, Tuul M, Zhao Y, Ando K, Saeki H, Oki E, Ohga T, Kitao H, Kakeji Y and Maehara Y: Prognostic relevance of KRAS and BRAF mutations in japanese patients with colorectal cancer. Int J Clin Oncol 18: 1042-1048, 2013.

27. Pang NK, Nga ME, Chin SY, Ismail TM, Lim GL, Soong R and Salto-Tellez M: KRAS and BRAF mutation analysis can be reliably performed on aspirated cytological specimens of metastatic colorectal carcinoma. Cytopathology 22: 358-364, 2011.

28. Moroni M, Veronese S, Benvenuti S, Marrapese G, Sartore-Bianchi A, Di Nicolantonio F, Gambacorta M, Siena S and Bardelli A: Gene copy number for epidermal growth factor receptor (EGFR) and clinical response to antiEGFR treatment in colorectal cancer: A cohort study. Lancet Oncol 6: 279-286, 2005.

29. Brevet M, Johnson ML, Azzoli CG and Ladanyi M: Detection of EGFR mutations in plasma DNA from lung cancer patients by mass spectrometry genotyping is predictive of tumor EGFR status and response to EGFR inhibitors. Lung Cancer 73: 96-102, 2011.

30. Ruhe JE, Streit S, Hart S, et al: Genetic alterations in the tyrosine kinase transcriptome of human cancer cell lines. Cancer Res 67: 11368-11376, 2007.

31. Di Nicolantonio F, Martini M, Molinari F, et al: Wild-type $\mathrm{BRAF}$ is required for response to panitumumab or cetuximab in metastatic colorectal cancer. J Clin Oncol 26: 5705-5712, 2008.

32. Frattini M, Saletti P, Romagnani E, Martin V, Molinari F, Ghisletta M, Camponovo A, Etienne LL, Cavalli F and Mazzucchelli L: PTEN loss of expression predicts cetuximab efficacy in metastatic colorectal cancer patients. Br J Cancer 97: $1139-1145,2007$. 
33. Molinari F, Felicioni L, Buscarino M, et al: Increased detection sensitivity for KRAS mutations enhances the prediction of anti-EGFR monoclonal antibody resistance in metastatic colorectal cancer. Clin Cancer Res 17: 4901-4914, 2011.

34. Lièvre A, Bachet JB, Le Corre $\mathrm{D}$, et al: KRAS mutation status is predictive of response to cetuximab therapy in colorectal cancer. Cancer Res 66: 3992-3995, 2006.

35. Heinemann V, Stintzing S, Kirchner T, Boeck S and Jung A Clinical relevance of EGFR- and KRAS-status in colorecta cancer patients treated with monoclonal antibodies directed against the EGFR. Cancer Treat Rev 35: 262-271, 2009.

36. Zlobec I, Bihl MP, Schwarb H, Terracciano L and Lugli A: Clinicopathological and protein characterization of BRAF- and K-RAS-mutated colorectal cancer and implications for prognosis. Intl J Cancer 127: 367-380, 2010.
37. Chaiyapan W, Duangpakdee P, Boonpipattanapong T, Kanngern S and Sangkhathat S: Somatic mutations of K-ras and BRAF in Thai colorectal cancer and their prognostic value. Asian Pac J Cancer Prev 14: 329-332, 2013.

38. Wu CM, Tang R, Wang JY, Changchien CR and Hsieh LL: Frequency and spectrum of K-RAS codons 12 and 13 mutations in colorectal adenocarcinomas from Taiwan. Cancer Genet Cytogenet 158: 55-60, 2005.

39. Kim ST, Park KH, Kim JS, Shin SW and Kim YH: Impact of kras mutation status on outcomes in metastatic colon cancer patients without anti-epidermal growth factor receptor therapy. Cancer Res Treat 45: 55-62, 2013.

40. Gallimore AM and Godkin A: Epithelial barriers, microbiota and colorectal cancer. N Engl J Med 368: 282-284, 2013. 